\title{
ORBITS OF CREATIVE SUBSPACES
}

\author{
R. G. DOWNEY
}

\begin{abstract}
It is shown that the creative r.e. subspaces fall into infinitely many distinct elementary classes. The techniques also extend to give some new results about orbits of creative subspaces and subfields in $L^{*}\left(V_{\infty}\right)$ and $L^{*}\left(F_{\infty}\right)$ respectively. Finally within each of these new elementary classes we construct infinitely many further orbits in the automorphism group of $L\left(V_{\infty}\right)$.
\end{abstract}

1. Introduction. We assume that the reader is already familiar with $L\left(V_{\infty}\right)$, the lattice of r.e. subspaces, and (for one result) its subsequent generalization to Steinitz closure systems as exposited in, for example, [MN1, MN2, NR1, NR2, Gu]. Recall that $V \in L\left(V_{\infty}\right)$ is called creative with productive function $f$ if $f$ is partial recursive, $\operatorname{dim}\left(V_{\infty} / V\right)=\infty$, and if $W_{e} \cap V=\{\overrightarrow{0}\}$ then $f(e) \in V_{\infty}-\left(W_{e} \oplus V\right)$ for all $W_{e} \in L\left(V_{\infty}\right)$. This definition from [MN1] is the obvious analogue of a creative set. Now Myhill's theorem [My] states that any pair of creative sets differ by a recursive permutation of $\omega$. The analogous statement in $L\left(V_{\infty}\right)$ fails by, for example, [MN1, Corollary 6.9]: There are creative subspaces $C_{1}, C_{2}$ such that no recursive automorphism $F$ of $L\left(V_{\infty}\right)$ takes $C_{1}$ to $C_{2}$. Indeed, in view of Guichard's $[\mathbf{G u}]$ classification of the automorphisms of $L\left(V_{\infty}\right)$ as those induced by recursive invertible semilinear transformations of $V_{\infty}$, it follows that $C_{1}$ and $C_{2}$ of [MN1, Corollary 6.9] are not even in the same orbit.

The purpose of this paper is to prove some significantly stronger results. For $L\left(V_{\infty}\right)$ we show the creative subspaces fall into infinitely many distinct elementary classes. To do this we introduce a new variety of creative subspace, creative of type $n$ (for $n \in \omega$ ), such that for each $n$ there is a formula $\Phi_{n}$ satisfied by subspaces which are creative of type $n$, but not of type $m$ for $m \neq n$.

Our next results concern automorphisms of $L^{*}\left(V_{\infty}\right)$, that is, $L\left(V_{\infty}\right)$ modulo finite dimensional subspaces. Very little is known about automorphisms of $L^{*}\left(V_{\infty}\right)$. Our results enable us to show that there are creative subspaces $C_{1}, C_{2}$ such that no automorphism of $L^{*}\left(V_{\infty}\right)$ takes $C_{1}$ to $C_{2}$ and indeed, $C_{1}$ and $C_{2}$ again fall into different elementary classes.

We extend some of these results to the general setting of a Steinitz closure system. This includes, for example, $L\left(F_{\infty}\right)$, the lattice of r.e. algebraically closed fields.

Finally we construct infinitely many orbits of the automorphism group of $L\left(V_{\infty}\right)$ within each of our new classes.

Received by the editors February 8, 1985.

1980 Mathematics Subject Classification (1985 Revision). Primary 03D45.

Research partially supported by N.U.S. Grant RP 85/83 (Singapore).

(c) 1987 American Mathematical Society $0002-9939 / 87 \$ 1.00+\$ .25$ per page 


\section{Results.}

(2.1) Definition. Let $V \in L\left(V_{\infty}\right)$. We say $V$ is creative of type $n$ if

(i) $V$ is creative,

(ii) there is a decidable $D \in L\left(V_{\infty}\right)$ such that $V \subseteq D$ and $\operatorname{dim}\left(V_{\infty} / D\right)=n$,

(iii) for all decidable subspaces $Q$, if $Q \supseteq V$ then $Q \supseteq D$.

REMARK. We remark that there is some mild conflict with the terminology of [MN1]. In keeping with subsequent papers, "recursive = recursive as a set" and "decidable = complemented" (cf. [NR3]).

(2.2) TheOREM. Let $n \in \omega$. There exists $V \in L\left(V_{\infty}\right)$ such that $V$ is creative of type $n$.

ProOF. We build an independent r.e. set $Q=\bigcup_{s} Q_{s}$ in stages so that $V=(Q)^{*}$, and a partial recursive function $f$ so that we meet the requirements

$$
P_{e}: W_{e} \cap V=\{\overrightarrow{0}\} \text { implies } f(e) \downarrow \text { and } f(e) \notin\left(W_{e} \oplus V\right) \text {. }
$$

We must also ensure that $\operatorname{dim}\left(V_{\infty} / V\right)=\infty$. This makes $V$ creative. Now let $B=\left\{a_{0}<a_{1}<\cdots\right\}$ list in order a recursive basis of $V_{\infty}$. Let $n$ be given. Let $D=\left\{d_{0}, d_{1}, \ldots\right\}$, where $d_{i}=a_{n+i}$ for all $i$. Let $K_{e}$ be the eth r.e. independent r.e. set such that $\operatorname{supp}_{B}(x) \subseteq D$ for all $x \in K_{e, s}$. Here $\operatorname{supp}_{B}(x)$ denotes the support of $x$ relative to $B$. Now form $J_{e}$ as follows: set $J_{e, s}=K_{e, t(s)}$, where

$$
t(s)=\max _{t \leq s}\left\{\forall i \leq t\left(d_{i} \in\left(K_{e, s}\right)^{*}\right)\right\} .
$$

Observe that $\operatorname{card}\left(J_{e}\right)=\infty \mathrm{iff}\left(K_{e}\right)^{*}=(D)^{*}$. To ensure (ii) and (iii) of (2.1) we meet the requirements

$$
R_{\langle e, y\rangle}: y \in J_{e} \text { and } \operatorname{card}\left(J_{e}\right)=\infty \text { implies that for some } x \in V, y \in \operatorname{supp}(e, x),
$$

where $\operatorname{supp}(e, x)$ denotes the support of $x$ relative to $J_{e}$. We say $R_{\langle e, y\rangle}$ is satisfied at state $s$ if there exists $z \in\left(Q_{s}\right)^{*}$ with $z \in\left(J_{e, s}\right)^{*}$ such that $y \in \operatorname{supp}(e, z)$. Notice that $z \in\left(J_{e, s}\right)^{*}$ means $\operatorname{supp}(e, y)$ is well defined. We meet the $R_{\langle e, y\rangle}$ by witnesses, which we denote by $x(e, y)$, and define at stage 0 . We say $R_{\langle e, y\rangle}$ requires attention at stage $s+1$ if

(i) $R_{\langle e, y\rangle}$ is not satisfied at stage $s$,

(ii) $y \in J_{e, s}$,

(iii) $x(e, y) \in\left(J_{e, s}\right)^{*}$.

Finally, in the construction we have a set $M$ which will witness that $\operatorname{dim}\left(V_{\infty} / V\right)=$ $\infty$.

\section{CONSTRUCTION.}

Stage 0. For all $e \in \omega$ set $f(e)=d_{3 e+1}$. Set $M=\left\{d_{3 e}: e \in \omega\right\}$ and $P=$ $M \cup\{f(e): e \in \omega\}$.

We now perform the first stage of the construction by defining the $x(e, y)$. It is simplest to view this as a subconstruction, as follows.

Subconstruction, stage 0 . Find $k(0)$ to be the least $k$ such that for $0=\langle e, y\rangle$, $\left\{y+d_{3 k+2}, d_{3 k+2}\right\} \cap(P)^{*}=\varnothing$. Set $x(e, y)=d_{3 k+2}$, and $E_{0}=P \cup\left\{y+d_{3 k+2}, d_{3 k+2}\right\}$.

Subconstruction, stage $s+1$. Find $k(s+1)$ to be the least $k^{\prime}>k(s)$ such that for $s+1=\left\langle e^{\prime}, y^{\prime}\right\rangle$

$$
\left\{y^{\prime}+d_{3 k^{\prime}+2}, d_{3 k^{\prime}+2}\right\} \cap\left(E_{s}\right)^{*}=\varnothing .
$$

Set $x\left(e^{\prime}, y^{\prime}\right)=d_{3 k^{\prime}+2}$ and $E_{s+1}=E_{s} \cup\left\{y^{\prime}+d_{3 k^{\prime}+2}, d_{3 k^{\prime}+2}\right\}$. 
This completes the subconstruction. Notice each stage is effective since $P$ is decidable and by dimension arguments we can find $k(s)$. The sequence $k(0), k(1), \ldots$ has the following key property.

(2.3) Let $z(0), z(1), \ldots$ be any sequence of integers with $z(i) \in\left\{y(i)+d_{k(i)+2}\right.$, $\left.d_{k(i)+2}\right\}$ with $i=\langle e(i), y(i)\rangle$; so $z(i) \in\{y(i)+x(e(i), y(i)), x(e(i), y(i))\}$. Then $P \cup\{z(i): i \in \omega\}$ is an independent set.

We return to the main construction.

Stage $s+1$.

Step 1. Find the least $\langle e, y\rangle \leq s$ (if any) such that $R_{\langle e, y\rangle}$ requires attention. If no such $\langle e, y\rangle$ exists, set $Q(s)=Q_{s}$, and go to Step 2. If $\langle e, y\rangle$ exists, there are two cases.

Case 1. $y \in \operatorname{supp}(e, x(e, y))$. Set $Q(s)=Q_{s} \cup\{x(e, y)\}$.

Case 2. $y \notin \operatorname{supp}(e, x(e, y))$. Set $Q(s) \cup\{y+x(e, y)\}$. (Notice that since $y \notin$ $\operatorname{supp}(e, x(e, y)), y \in \operatorname{supp}(e, y+x(e, y))$ and hence $R_{\langle e, y\rangle}$ is now satisfied.)

Step 2. Find the least $e \leq s$ (if any) such that $f(e) \in(Q(s))^{*} \oplus\left(W_{e, s}\right)^{*}$ and $(Q(s))^{*} \cap\left(W_{e, s}\right)^{*}=\{\overrightarrow{0}\}$. If such an $e$ exists set $Q_{s+1}=Q(s) \cup\{f(e)\}$. Otherwise set $Q_{s+1}=Q(s)$.

Now set $Q=\bigcup_{s} Q_{s}$ and $V=(Q)^{*}$.

END OF CONSTRUCTION.

VERIFICATION. Evidently each $P_{e}$ or $R_{e}$ receives attention at most once and is met by this action. For the $R_{e}$ this is obvious. In the $P_{e}$ case, if $f(e) \in(Q(s))^{*} \oplus$ $\left(W_{e, s}\right)^{*},(Q(s))^{*} \cap\left(W_{e, s}\right)^{*}=\{\overrightarrow{0}\}$, and $P_{e}$ receives attention, we add $f(e)$ to $Q_{s+1}-$ $Q(s)$. Now $f(e)=q+w$ where $q \in(Q(s))^{*} w \in\left(W_{e, s}\right)^{*}$, and $w \neq \overrightarrow{0}$ by construction and (2.3). Hence $w \in\left(Q_{s+1}^{*} \cap\left(W_{e, s}\right)^{*}\right.$.

It is easy to see that all the $P_{e}$ and $R_{\langle e, y\rangle}$ are met, and that $(M)^{*} \cap V=\{\overrightarrow{0}\}$ by (2.3) and selection of $f(e)$. Thus $V$ is creative, and since when $R_{\langle e, y\rangle}$ receives attention some $z \in(D)^{*}$ is put into $Q$, it follows that $Q \subseteq(D)^{*}$.

Finally, let $H$ be decidable and suppose $H \supseteq V$. Let $H^{\prime}=H \cap(D)^{*}$. Then $H^{\prime}$ is decidable since the intersection of two decidable subspaces is decidable (cf. [AD]). Now if $H \nsupseteq(D)^{*}$, then $V \subseteq H^{\prime} \nsubseteq(D)^{*}$. Let $R$ be a recursive basis of $H^{\prime}$. Extend $R$ to a recursive basis $T$ of $(D)^{*}$ so that $T=R \cup G$ with $G \neq \varnothing$. Let $y \in G$. By the $s-m-n$ theorem let $K_{e}=T$. Now $\operatorname{card}\left(J_{e}\right)=\infty$ since $K_{e}$ is a basis of $(D)^{*}$. Hence $R_{\langle e, y\rangle}$ receives attention. In either case some $z \in\{y+x(e, y), x(e, y)\}$ is put into $V$ and furthermore $y \in \operatorname{supp}(e, z)$. But then it cannot be that $V \subseteq H^{\prime} \subseteq\left(J_{e}-\{y\}\right)^{*}$. Therefore if $H$ is decidable and $H \supseteq V$, then $H \supseteq D$.

(2.4) COROLLARY. There are infinitely many distinct elementary classes of creative subspaces.

Proof. Consider $\left\{C_{i}\right\}_{i \in \omega}$, where $C_{i}$ is creative of type $i$.

Now let $[V]$ denote the equivalence class of $V \in L\left(V_{\infty}\right)$ under $=^{*}$, where $V={ }^{*}$ $W$ iff for some finite set $F,(V \cup F)^{*}=(W \cup F)^{*}$. Recall $L^{*}\left(V_{\infty}\right)=L\left(V_{\infty}\right)$ $\bmod =^{*}$. We have

(2.5) COROllaRY. There exist creative subspaces $C_{1}, C_{2}$ such that $\left[C_{1}\right]$ and $\left[C_{2}\right]$ are in different elementary classes $\left(\right.$ for $\left.L^{*}\left(V_{\infty}\right)\right)$. 
ProOF. Let $V$ be as constructed in (2.1). Let $W=(C)^{*}$, where $C$ is an r.e. creative subset of a recursive basis. As in $[\mathbf{M N 1}],(C)^{*}$ is creative. In $[\mathbf{A D}]$ it is observed that $(C)^{*}$ has the property that given any decidable $D$ with $(C)^{*} \subseteq D$, there exists a decidable $D^{\prime}$ with $(C)^{*} \subseteq D^{\prime} \subseteq D$ and $\operatorname{dim}\left(D / D^{\prime}\right)-\infty$. Clearly $C_{1}=V$ and $C_{2}=W$ suffice.

Actually, Corollary (2.5) may be deduced from the following result of Remmel (in [NR3]): There is a simple subset of a recursive basis generating a creative subspace. To see this, we shall say a creative subspace $V$ has type $<\omega$ if $V$ is not of type $n$ for any $n$, but if $D$ is decidable and $D \supset V$ then $\operatorname{dim}(V / D)<\infty$. We have

(2.6) THEOREM. Let $S$ be a simple subset of a recursive basis $B$ of $V$, generating a creative subspace. Then $(S)^{*}$ is of type $<\omega$.

Proof. Obviously $(S)^{*}$ is not of type $n$ for any $n$. Now, let $D$ be decidable with $(S)^{*} \subseteq D$ and $\operatorname{dim}\left(V_{\infty} /(D)^{*}\right)=\infty$. Form a cobasis $B^{\prime}=\left\{b_{0}, b_{1}, \ldots\right\}$ as follows. Let $b_{0}=\mu y(y \in B$ and $y \notin D)$. Let $b_{s}=\mu y\left(y \in B\right.$ and $\left.y \notin\left(D \cup\left\{b_{j}: j<s\right\}\right)^{*}\right)$. If $\operatorname{dim}\left(V_{\infty} / D\right)=\infty$, then $B^{\prime}$ is an infinite recursive subset of $B$ (as $D$ is decidable). Evidently, as $S \subseteq D, S \cap B^{\prime}=\varnothing$. This contradicts the simplicity of $S$ in $B$.

Thus we can see that $(S)^{*}$ cannot be automorphic with $(C)^{*}$ of $(2.5)$ in either $L\left(V_{\infty}\right)$ or $L^{*}\left(V_{\infty}\right)$. It is also possible to modify our technique to construct yet another orbit: we can also construct a creative $V \in L\left(V_{\infty}\right)$ such that there is a decidable $D$ with $V \subseteq D, \operatorname{dim}\left(V_{\infty} / D\right)=\infty$, and for all decidable $D^{\prime} \supseteq V, D^{\prime} \supseteq D$. We leave this modification to the reader. We ask if there are any other elementary classes or orbits of creative subspaces in $L^{*}\left(V_{\infty}\right)$ apart from these three types.

Before continuing, we would like to address some comments to generalizations of these results to abstract dependence settings. We assume the reader is already familiar with the setting of a recursive Steinitz closure system, and the analogous definition of creativity there (cf. [NR1,2, MN2]). For the reader familiar with this setting, we state the following results which can be obtained by standard modification of our arguments.

(2.6) THEOREM. Let $(M, \mathrm{cl})$ be a recursive Steinitz closure system satisfying either Axiom I or Axiom II below. Then there exist creative $V_{1}, V_{2} \in L(M)$ such that $V_{1}$ and $V_{2}$ lie in different elementary clasess in either $L(M)$ or $L^{*}(M)$.

Axiom I. If $I$ is an infinite independent set and $n \in \omega$, there exists $y \in \operatorname{cl}(I)$ such that card $\left(\operatorname{supp}_{I}(y)\right) \geq n$.

Axiom II (Nerode and Remmel [NR1,2]). If $I$ is an infinite set independent in $\left(M, \operatorname{cl}_{V}\right)$, where $V$ is closed, then in $\left(M, \operatorname{cl}_{V}\right)$ the dimension of $\operatorname{cl}(I \cup\{x\})-\operatorname{cl}(I)$ is infinite.

We remark that Axiom I suffices to get creative r.e. closed sets generated by simple subsets of recursive bases modifying Remmel's argument, and Axiom II suffices for getting type 0 creative r.e. closed sets using our argument. It is easy to construct a creative subset of a recursive basis generating a creative closed set, and so the analogue of (2.5) or (2.6) applies. Of course, we do not seem to get the full analogue (that is, for $n>0$ ) of $(2.1)$ since the proof relies heavily on the modularity of $L\left(V_{\infty}\right)$ which does not hold for $L\left(F_{\infty}\right)$. Whether or not (even) type 1 creative subfields exist is thus still open. 
For our final result, we return to $L\left(V_{\infty}\right)$. The natural question to ask is whether or not any of our new varieties of creative subspaces form orbits in the automorphism group of $L^{*}\left(V_{\infty}\right)$. For simplicity we concentrate on type $n$ creative subspaces. We shall need the following.

(2.8) THEOREM (GUICHARD $[\mathbf{G u}]$ ). Every automorphism of $L\left(V_{\infty}\right)$ is induced by a recursive invertible semilinear transformation.

We shall construct $C_{1}, C_{2}$, creative subspaces of type $n$, that are nonautomorphic by diagonalization over all recursive semilinear transformations. (It is easy to extend this to construct infinitely many nonautomorphic $C_{i}$.) From a technical point of view, we remark that this is a somewhat more delicate construction than the diagonalizations in [Gu, DH or NR2]. In those papers, nonautomorphic subspaces $V_{1}, V_{2}$ (of certain types) are constructed; but the diagonalizations in those papers actually ensure that no recursive permutation of $V_{\infty}$ (as a set) takes $V_{1}$ to $V_{2}$. This is not possible in our case since by Myhill's theorem [My] any pair of creative sets differ by a recursive permutation of $\omega$, and because TeKolste [MN1, Theorem 5.2] has shown that every creative subspace is a creative subset of $V_{\infty}$.

(2.9) THEOREM. Let $n \in \omega$. There exist creative $V, W \in L\left(V_{\infty}\right)$ such that no automorphism $\Phi$ of $L\left(V_{\infty}\right)$ takes $V$ to $W$.

Proof. We build $Q(V)=\bigcup_{s} Q_{s}(V)$ and $Q(W)=\bigcup_{s} Q_{s}(W)$ so that $V=$ $(Q(V))^{*}$ and $W=(Q(W))^{*}$ have the desired properties. The intention is that we read $Q()$ in place of $Q$ in the proof of (2.2). Thus we have requirements

$$
\begin{aligned}
& P_{e}^{V}: W_{e} \cap V=\{\overrightarrow{0}\} \text { implies } f(e) \downarrow \text { and } f(e) \notin\left(W_{e} \oplus V\right), \\
& P_{e}^{W}: W_{e} \cap W=\{\overrightarrow{0}\} \text { implies } f(e) \downarrow \text { and } f(e) \notin\left(W_{e} \oplus W\right),
\end{aligned}
$$

where we use the same $f(e)$ for both constructions, with $f(e)$ as in the proof of (2.2). We similarly have $R_{\langle e, y\rangle}^{V}$ and $R_{\langle e, y\rangle}^{W}$. Finally, we have the nonautomorphism requirements

$$
\begin{aligned}
N_{e}: & \text { If } \Phi_{e} \text { is a recursive invertible semilinear transformation, } \\
& \text { then for some } x, \text { either } x \in V \text { and } \Phi_{e}(x) \in W \text {, or } \\
& x \notin V \text { and } \Phi_{e}(x) \in W .
\end{aligned}
$$

Here $\left\{\Phi_{e}\right\}_{e \in \omega}$ is a list of all partial recursive semilinear transformations; that is, when defined, they are semilinear. This can be easily achieved by halting the enumeration of the eth partial recursive function when it becomes nonsemilinear on its currently defined domain.

Injury of requirements necessistates the use of a potentially infinite sequence of witnesses $\left\{x(e, y, s): s \in \omega^{\prime}\right\}$ in place of $x(e, y)$ for the satisfaction of the $R_{\langle e, y\rangle}^{V}$ and $R_{\langle e, y\rangle}^{W}$. Thus, $R_{\langle e, y\rangle}^{V}$ or $R_{\langle e, y\rangle}^{W}$ requires attention at stage $s+1$ if (i) and (ii) hold as before, and

(iii)' $x(e, y, s) \in\left(J_{e, s}\right)^{*}$.

It will be a feature of the construction that the same witnesses are used for both $\langle e, y\rangle$ requirements. Our priority ranking is

$$
R_{0}^{V}, R_{1}^{W}, N_{0}, R_{1}^{V}, R_{1}^{W}, \ldots
$$


For the sake of the $N_{e}$ we shall place markers $\Gamma(e, s), z(e, s)$, and $\Lambda(e, s)$ which may be defined or undefined. These are intended to witness the failure of $\Phi_{e}$ to be an automorphism. With the appropriate meaning, we say $\Phi_{e}$ is consistent at state $s$ if it currently believes (at stage $s$ ) that is a semilinear transformation taking $V_{s}$ to $W_{s}$ (that is, where it is defined). At stage $s$ we need only attack consistent $\Phi_{e}$ 's. We say $N_{e}$ requires attention at stage $s$ if $\Phi_{e}$ is consistent and either

(2.10) (i) $N_{e}$ is waiting, and

(ii) for some $x(e, y, s)$ with $\langle e, y\rangle>e+1$ there exists $z$ such that $\Phi_{e, s}(z) \downarrow$ and $\Phi_{e, s}(z)=x(e, y, s)$, or

(2.11) (i) $N_{e}$ is active, and

(ii) $\Phi_{e, s}(\Gamma(e, s)) \downarrow$ and $\Phi_{e, s}(\Gamma(e, s)+z(e, s)) \downarrow$.

CONSTRUCTION.

Stage 0. For all $e \in \omega$, set $f(e)=d_{3 e+1}, M=\left\{d_{3 e}: e \in \omega\right\}$, and $P=M \cup$ $\{f(e): e \in \omega\}$. Now define $x(e, y, 0)=d_{3 k+2}$, where $\langle e, y\rangle=0$ and $k$ is the least number with $\left\{y+d_{3 k+2}, d_{3 k+2}\right\}^{*} \cap \Lambda(P)^{*}=\{\overrightarrow{0}\}$. For all $g \in \omega$, declare $\Gamma(g, 0)$, $z(g, 0)$, and $\Lambda(g, 0)$ as undefined. (Our convention is they will stay undefined at any future stage unless some action is taken to define them.) Also declare $N_{g}$ as waiting.

Stage $s+1$.

Step 1 . Find the requirement $R$ of highest priority to require attention. If none exists, set $Q_{s}^{\prime}(V)=Q_{s}(V), Q_{s}^{\prime}(W)=Q_{s}(W)$ and go to Step 2. Otherwise, adopt the appropriate case below.

(2.12) $R=R_{\langle e, y\rangle}^{V}$. Cancel all $\Gamma(g, s), z(g, s)$, and $\Lambda(g, s)$ markers for all $g \geq$ $\langle e, y\rangle$, and declare $N_{g}$ as waiting. Set $Q_{s}^{\prime}(W)=Q_{s}(W)$. As before there are two cases: If $y \in \operatorname{supp}(e, x(e, y, s))$, set $Q_{s}^{\prime}(V)=Q_{s}(V) \cup\{x(e, y, s)\}$; otherwise, set $Q_{s}^{\prime}(W)=Q_{s}(V) \cup\{y+x(e, y, s)\}$.

(2.13) $R=R_{\langle e, y\rangle}^{W}$. Same as (2.12) with the roles of $V$ and $W$ reversed.

(2.14) $R=N_{e}$. Cancel all $\Gamma(g, s), z(g, s)$, and $\Lambda(g, s)$ markers for all $g>e$, and declare $N_{g}$ as waiting. Now there are several subcases.

Case 1. (2.10) holds. Mark $z$ and $x(e, y, s)$ by setting $z(e, s+1)=z$ and $\Lambda(e, s+1)=x(e, y, s)$.

Subcase (a). $z \notin\left(Q_{s}(V) \cup\{f(e): e \in \omega\}\right)^{*}$. In this case, set $Q_{s}^{\prime}(V)=Q_{s}(V)$ and set $Q_{s}^{\prime}(W)=Q_{s}(W) \cup\{x(e, y, s)\}$. Notice this makes $\Phi_{e}$ no longer consistent. Go to Step 2.

Subcase (b). $z \in\left(Q_{s}(V) \cup\{f(e): e \in \omega\}\right)^{*}$. In this case, declare $N_{e}$ as active. Now find the least $k$ such that

$$
\begin{aligned}
& \left\{z(e, s)+d_{3 k+2}, d_{3 k+2}\right\} \\
& \cap\left[Q_{s}(V) \cup Q_{s}(W) \cup P \cup\{x(f, p, s):\langle f, p\rangle \leq e\}\right. \\
& \cup\{z(g, s): g \leq e\} \cup\{\Lambda(g, s): g \leq e\} \cup\{\Gamma(g, s): g \leq e\}]^{*}=\varnothing .
\end{aligned}
$$

Set $\Gamma(e, s+1)=d_{3 k+2}, Q_{s}^{\prime}(V)=Q_{s}(V), Q_{s}^{\prime}(W)=Q_{s}(W)$, and to to Step 2 .

Case 2. (2.11) holds. There are two subcases.

Subcase (a). $\Phi_{e, s}(\Gamma(e, s)) \notin\left(Q_{s}(W) \cup\{f(e): e \in \omega\}\right)^{*}$. In this case set $Q_{s}^{\prime}(V)=$ $Q_{s}(V) \cup\{\Gamma(e, s)\}$. Notice this temporarily satisfies $N_{e}$ since $\Gamma(e, s)$ is outside of $V$ and is being taken into $W$ by $\Phi_{e}$. Go to Step 2 .

Subcase (b). $\Phi_{e, s}(\Gamma(e, s)) \in\left(Q_{s}(W) \cup\{f(e): e \in \omega\}\right)^{*}$. Now as $N_{e}$ is active, by Case 1 (b) we also know that $z(e, s) \in\left(Q_{s}(V) \cup\{f(e): e \in \omega\}\right)^{*}$, and hence by the 
way we chose $\Lambda(e, s), \lambda_{1} \Lambda(e, s)+\lambda_{2} \Phi_{e, s}(\Gamma(e, s)) \notin\left(Q_{s}(V) \cup\{f(e): e \in \omega\}\right)^{*}$ for $\lambda_{1}, \lambda_{2} \neq 0$. (This will follow by induction in the verification.) Now it follows that we can temporarily satisfy $N_{e}$ by setting $Q_{s}^{\prime}(V)=Q_{s}(V) \cup\{z(e, s)+\Gamma(e, s)\}$ and $Q_{s}^{\prime}(W)=Q_{s}(W)$, and not adding $\Phi_{e, s}(\Gamma(e, s)+z(e, s))=\lambda \Lambda(e, s)+\mu \Phi_{e, s}(\Gamma(e, s))$ to $Q(W)$, which is achieved by Step 3 and Step 1, Case 1, Subcase (b).

Step 2. Find the least $e \leq s$ (if any) such that $f(e) \in\left(Q_{s}^{\prime}(V)\right)^{*} \oplus\left(W_{e, s}\right)^{*}$ with $Q_{s}^{\prime}(V)^{*} \cap\left(W_{e, s}\right)^{*}=\{\overrightarrow{0}\}$. If no $e$ exists, set $Q_{s+1}(V)=Q_{s}^{\prime}(V)$. Otherwise, $\left.Q_{s+1}(V) \cup\{f(e))\right\}$. Now proceed similarly for $W$.

Step 3. Now we must redefine the $x(e, y, s+1)$ so as not to interfere with markers etc. (of higher priority). Let $E=\left(Q_{s+1}(V) \cup Q_{s+1}(W) \cup P \cup N\right)^{*}$, where $N$ is the collection of all markers, $x(e, y, s)$ and their $\Phi_{j}$ images for $j<s$ mentioned so far. Now, if in Step 1 no requirement received attention, for all $\langle e, y\rangle \leq s$ set $x(e, y, s+1)=x(e, y, s)$. Find the least $k$ such that for $\left\langle e^{\prime}, y^{\prime}\right\rangle=s+1,\left\{y^{\prime}+\right.$ $\left.d_{3 k+2}, d_{3 k+2}\right\} \cap(E)^{*}=\varnothing$. Set $x\left(e^{\prime}, y^{\prime}, s+1\right)=d_{3 k+2}$, and go to stage $s+2$.

Otherwise, some requirement $R$ received attention in Step 1. If $R=R_{e}^{V}$ or $R_{e}^{W}$, set $m(s+1)=e$. If $R=N_{e}$, set $M(s+1)=e+1$. We now generate $x(f, g, s+1)$ inductively in substages $j$ for $m(s+1) \leq j \leq s+1$. Set $F_{0}=E$.

Substage $j$. Find the unique $(e, y)$ with $\langle e, y\rangle=j$. Now find the least $k(j)$ with $\left\{y^{\prime}+d_{3 k(j)+2^{\prime}}, d_{3 k(j)+2}\right\} \cap\left(F_{j}\right)^{*}=\varnothing$. Set $x(e, y, s+1)=d_{3 k(j)+2}$. If $j=s+1$ go to stage $s+2$. Otherwise, set $F_{j+1}=F_{j} \cup\left\{y^{\prime}+x(e, y, s+1), x(e, y, s+1)\right\}$ and go to substage $j+1$.

END OF CONSTRUCTION.

We now sketch the verification. We need a simultaneous induction for all the $R_{e}^{V}$, $R_{e}^{W}$, and $N_{e}$ and to show that $\lim _{s} x(e, y, s)=x(e, y)$ exists. It is clear that Step 3 ensures that if $t_{0}$ is the least stage by which a requirement of priority higher than $R_{\langle e, y\rangle}^{V}$ receives attention, then by Step 3 of the construction, $x(e, y, s)$ can be reset at most twice more: once for $R_{\langle e, y\rangle}^{V}$ and once for $R_{\langle e, y\rangle}^{W}$ (since $N_{j}$ and $R_{j} j \geq\langle e, y\rangle$, cannot interfere with $x(e, y, s)$ by the way we define $m(s+1))$. Thus it suffices to prove that the $N_{e}$ receives attention at most finitely often and is met, and then a proof similar to that of (2.1) will do the rest. The key point is that if all requirements $R$ of higher priority than $N_{e}$ receive attention for the last time at stage $t_{1}$, then if $\Phi_{e}$ really is a recursive invertible semilinear transformation (2.10) must apply at some stage $s_{1}>t_{1}$. At stage $s_{1}, \Lambda\left(e, s_{1}\right)$ and $j\left(e, s_{1}\right)$ become defined and by Step 3 , the $x\left(g, y, s_{1}\right)$ for $\langle g, y\rangle>e$ are all reset so that their activity cannot interfere with these markers. In particular if Subcase (a) applies, $x\left(e, s_{1}\right)$ is permanently restrained from $(Q(V) \cup\{f(e): e \in \omega\})^{*}$; by Step 3 if $q \in Q_{r, s}-\left(Q_{s_{1}}(V) \cup\{f(e): e \in \omega\}\right)^{*}$, then $q=x(g, y, s)$ or $q=x(g, y, s)+y$ for $\langle g, y\rangle>e$ and so by Step 3 is independent of $\left(Q_{s}(V) \cup\{f(e): e \in \omega\} \cup\left\{z\left(e, s_{1}\right)\right\}\right)^{*}$. Thus in Subcase $(\mathrm{a}), z\left(e, s_{1}\right) \notin V$, and $\Phi_{e}\left(z\left(e, s_{1}\right)\right)=\Lambda\left(e, s_{1}\right)$ with $\Lambda\left(e, s_{1}\right) \in W$.

In Subcase (b), we define $\Gamma\left(e, s_{1}\right)$ and again in Step 3 reset the $x\left(g, y, s_{1}\right)$ to not interfere with $z\left(e, s_{1}\right)+\Gamma\left(e, s_{1}\right)$ or $\Gamma\left(e, s_{1}\right)$, or with $\Lambda\left(e, s_{1}\right)$. Thus when Case 2 occurs as we observed in the construction, we diagonalize forever $\Phi_{e}$ from being a candidate, because $\Phi_{e}$ will no longer be consistent. The result now follows by induction, and the arguments of (2.1).

With similar arguments it is possible to construct orbits within other classes of creative subspaces. We leave these to the reader. One final remark which we feel is relevant to this topic is the following: By taking Remmel's construction in [NR3] 
of a simple subset of a recursive basis generating a creative subspace, and blending it with Martin's construction of [So, Chapter X, Exercise 5.5], it is possible to construct a simple subset $S$ of a recursive basis $B$ contained in no maximal subset of $B$, with $(S)^{*}$ creative. We ask if such a subspace is contained in a maximal subspace and, in general, its every creative subspace contained in a maximal (or $h h$-simple) subspace. We believe this may be relevant to orbits of creative subspaces for $L^{*}\left(V_{\infty}\right)$.

ADDED IN PROOF. Jeff Remmel and the author have solved this last question by constructing atomless creative subspaces.

\section{REFERENCES}

[AD] C. J. Ash and R. G. Downey, Decidable subspaces and recursively enumerable subspaces, J. Symbolic Logic 49 (1984), 1137-1145.

[Ba] J. Baldwin, First order theories of abstract dependence relations, Ann. Pure Appl. Logic 26 (1984), 215-243.

[Do] R. G. Downey, Bases of supermaximal subspaces and Steinitz systems. II, Z. Math. Logik Grundlagen Math. (to appear).

[DH] R. G. Downey and G. R. Hird, Automorphisms of supermaximal subspaces, J. Symbolic Logic 50 (1985), 1-9.

[Gu] D. Guichard, Automorphisms of substructure lattices in effective algebra, Ann. Pure Appl. Logic 25 (1983), 47-58.

[KR] I. Kalantari and A. Retzlaff, Maximal vector spaces under automorphisms of the lattice of recursively enumerable vector spaces, J. Symbolic Logic 42 (1977), 481-491.

[MN1] G. Metakides and A. Nerode, Recursively enumerable vector spaces, Ann. Math. Logic 11 (1977), 147-171.

[MN2] - Recursion theory on fields and abstract dependence, J. Algebra 65 (1980), 36-59.

[My] J. R. Myhill, Creative sets, Z. Math. Logik Grundlagen Math. 1 (1955), 97-108.

[NR1] A. Nerode andd J. B. Remmel, Recursion theory of matroids, Patras Logic Symposion (Ed., G. Metakides), North-Holland, Amsterdam, 1982, pp. 41-65.

[NR2] _ Recursion theory on matroids. II, Southeast Asian Conference on Logic (Eds., C. T. Chong and M. J. Wicks), North-Holland, Amsterdam, 1983, pp. 133-184.

[NR3] _ A survey of the lattices of r.e. substructures, Recursion Theory (Eds., A. Nerode and R. Shore), Proc. Sympos. Pure Math., vol. 43, Amer. Math. Soc., Providence, R.I., 1985, pp. 323-375.

[So] R. I. Soare, Recursively enumerable sets and degrees, Springer Verlag Omega series (to appear).

Department of MAthematics, Victoria University of Wellington, Private BAG, WeLlington, NeW ZEALAND 